\title{
The AGILE monitoring of Cygnus X-3: transient gamma-ray emission and spectral constraints
}

\author{
G. Piano ${ }^{1,2}$, M. Tavani ${ }^{1,2,3,8}$, V. Vittorini ${ }^{1}$, A. Trois ${ }^{9}$, A. Giuliani ${ }^{4}$, A. Bulgarelli ${ }^{5}$, Y. Evangelista ${ }^{1}$, P. Coppi ${ }^{15}$, \\ E. Del Monte ${ }^{1}$, S. Sabatini ${ }^{1,2,8}$, E. Striani ${ }^{3,8}$, I. Donnarumma ${ }^{1}$, D. Hannikainen ${ }^{16,17}$, K. I. I. Koljonen ${ }^{16}$, \\ M. McCollough ${ }^{18}$, G. Pooley ${ }^{19}$, S. Trushkin ${ }^{20}$, R. Zanin ${ }^{21}$, G. Barbiellini ${ }^{6}$, M. Cardillo ${ }^{1,3}$, P. W. Cattaneo ${ }^{7}$, \\ A. W. Chen ${ }^{4}$, S. Colafrancesco ${ }^{12,13}$, M. Feroci ${ }^{1}$, F. Fuschino ${ }^{5}$, M. Giusti ${ }^{1,2}$, F. Longo $^{6}$, A. Morselli ${ }^{8}$, A. Pellizzoni ${ }^{9}$, \\ C. Pittori ${ }^{11,12}$, G. Pucella ${ }^{10}$, M. Rapisarda ${ }^{10}$, A. Rappoldi ${ }^{7}$, P. Soffitta ${ }^{1}$, M. Trifoglio ${ }^{5}$, \\ S. Vercellone ${ }^{14}$, and F. Verrecchia ${ }^{11,12}$ \\ (Affiliations can be found after the references)
}

Received 1 March 2012 / Accepted 26 July 2012

\begin{abstract}
We present the AGILE-GRID (Astro-rivelatore Gamma a Immagini LEggero - Gamma-Ray Imaging Detector) monitoring of Cygnus X-3, during the period between November 2007 and July 2009. We report here the whole AGILE-GRID monitoring of Cygnus X-3 in the AGILE "pointing" mode data-taking, to confirm that the $\gamma$-ray activity coincides with the same repetitive pattern of multiwavelength emission and analyze in depth the overall $\gamma$-ray spectrum by assuming both leptonic and hadronic scenarios. Seven intense $\gamma$-ray events were detected in this period, with a typical event lasting one or two days. These durations are longer than the likely cooling times of the $\gamma$-ray emitting particles, implying we see continuous acceleration rather than the result of an impulsive event such as the ejection of a single plasmoid that then cools as it propagates outwards. Cross-correlating the AGILE-GRID light curve with both X-ray and radio monitoring data, we find that the main events of $\gamma$-ray activity were detected while the system was in soft spectral X-ray states (RXTE/ASM (Rossi X-ray Timing Explorer/All-Sky Monitor) count rate in the 3-5 keV band $\gtrsim 3$ counts $^{-1}$ ), that coincide with local and often sharp minima of the hard X-ray flux (Swift/BAT (Burst Alert Telescope) count rate $\lesssim 0.02$ counts $\mathrm{cm}^{-2} \mathrm{~s}^{-1}$ ), a few days before intense radio outbursts. This repetitive temporal coincidence between the $\gamma$-ray transient emission and spectral state changes of the source turns out to be the spectral signature of $\gamma$-ray activity from this microquasar. These $\gamma$-ray events may thus reflect a sharp transition in the structure of the accretion disk and its corona, which leads to a rebirth of the microquasar jet and subsequent enhanced activity in the radio band. The $\gamma$-ray differential spectrum of Cygnus X-3 (100 MeV-3 GeV), which was obtained by averaging the data collected by the AGILE-GRID during the $\gamma$-ray events, is consistent with a power law of photon index $\alpha=2.0 \pm 0.2$. Finally, we examine leptonic and hadronic emission models for the $\gamma$-ray events and find that both scenarios are valid. In the leptonic model - based on inverse Compton scatterings of mildly relativistic electrons on soft photons from both the Wolf-Rayet companion star and the accretion disk - the emitting particles may also contribute to the overall hard X-ray spectrum, possibly explaining the hard non-thermal power-law tail seen during special soft X-ray states in Cygnus X-3.
\end{abstract}

Key words. stars: individual: Cygnus X-3 - X-rays: binaries - radio continuum: general - radiation mechanisms: non-thermal stars: winds, outflows - gamma rays: general

\section{Introduction}

Cygnus X-3 is the brightest radio source among all known microquasars and was discovered, as an X-ray source, in 1966 (Giacconi et al. 1967). It is a high-mass X-ray binary, whose companion star is a Wolf-Rayet (WR) star (van Kerkwijk et al. 1992) with a strong helium stellar wind (Szostek \& Zdziarski 2008). The system is located at a distance of about 7-10 kpc (Bonnet-Bidaud \& Chardin 1988; Ling et al. 2009). The orbital period is $4.8 \mathrm{~h}$, as inferred from infrared (Becklin et al. 1973), X-ray (Parsignault et al. 1972), and $\gamma$-ray (Abdo et al. 2009) observations. Owing to its very tight orbit (orbital distance $d \approx 3 \times 10^{11} \mathrm{~cm}$ ), the compact object is totally enshrouded in the wind of the companion star ${ }^{1}$. The nature of the compact object

\footnotetext{
The observational evidence of this strong wind can be found in the prominent attenuation of the Cygnus X-3 power density spectrum (PDS) for frequencies above $0.1 \mathrm{~Hz}$ (Axelsson et al. 2009; Koljonen et al. 2011)
}

is still uncertain ${ }^{2}$ (Vilhu et al. 2009), although a black hole scenario is favored (Szostek \& Zdziarski 2008; Szostek et al. 2008). In the radio band, the system shows strong flares ("major radio flares") reaching up to few tens of Jy. Radio observations at milliarcsec scales confirm emissions (at $\mathrm{cm}$ wavelengths) from both a core and a one-sided relativistic jet $(v \sim 0.81 c)$, with an inclination to the line-of-sight of $\lesssim 14^{\circ}$ (Mioduszewski et al. 2001). The radiation from the jet dominates the radio emission from the core during (and soon after) the major flares (Tudose et al. 2010).

Cygnus X-3 exhibits a clear, repetitive pattern of (anti)correlations between radio and X-ray emission, and an overall anticorrelation between soft and hard X-ray fluxes (McCollough et al. 1999; Szostek et al. 2008). The most important pattern of correlations found by Szostek et al. (2008)

2 Published results suggest either a neutron star of $1.4 M_{\odot}$ (Stark \& Saia 2003) or a black hole with a mass $\lesssim 10 M_{\odot}$ (Hanson et al. 2000; Shrader et al. 2010). 
is related to the connection between radio $(8.3 \mathrm{GHz}$ band, GBI) and soft X-ray emissions (3-5 keV band of the Rossi X-ray Timing Explorer/All-Sky Monitor (RXTE/ASM)). When the soft X-ray flux is above the transition level (3 counts/s), the source can be found in different states, depending on the level of the radio flux density. In particular, the quenched state is characterized by a radio flux density $\leqslant 30 \mathrm{mJy}$ and followed by a major-flaring state with values of radio flux density $\geqslant 1 \mathrm{Jy}$. It is very important to emphasize that all major radio flares have been observed after a quenched state, and in almost all cases the quenched state is followed by a major flare. After a major flare, a "hysteresis" in the radio/soft-X-ray plane is found, because the decline in the radio flux density never occurs by means of a quenched state.

Firm detections of high-energy $\gamma$-rays (HE $\gamma$-rays: $>100 \mathrm{MeV}$ ) from Cygnus X-3 ${ }^{3}$ were published at the end of 2009: the AGILE (Astro-rivelatore Gamma a Immagini LEggero) team found evidence that strong $\gamma$-ray transient emission above $100 \mathrm{MeV}$ coincided with special X-ray/radio spectral states (Tavani et al. 2009a), and the Fermi-LAT (Large Area Telescope) collaboration announced the detection of $\gamma$-ray orbital modulation (Abdo et al. 2009). The peak $\gamma$-ray isotropic luminosity detected above $100 \mathrm{MeV}$ is $L_{\gamma} \sim 10^{36} \mathrm{erg} \mathrm{s}^{-1}$ (for a distance of $7-10 \mathrm{kpc}$ ). The $\gamma$-ray emission is most likely associated with a relativistic jet (Tavani et al. 2009a; Abdo et al. 2009; Dubus et al. 2010; Cerutti et al. 2011; Zdziarski et al. 2012a), but the radiative process (leptonic or hadronic) is uncertain.

A possible leptonic scenario for $\gamma$-ray emission in Cygnus X-3 was proposed by Dubus et al. (2010): stellar ultraviolet (UV) photons are Compton upscattered to HE by relativistic electrons accelerated in the jet. The particle acceleration could take place in a shock where the jet interacts with the dense stellar wind of the WR star. The emerging picture is that of a jet with moderate bulk relativistic speed and oriented not too far from the line-of-sight.

The $\gamma$-ray modulation - coherent with the orbital period suggests that the emitting region is located at distances of between $\sim 10^{10} \mathrm{~cm}$ and $\sim 3 \times 10^{12} \mathrm{~cm}(10 d)$ from the compact object (Dubus et al. 2010; Cerutti et al. 2011). The lack of modulation at radio wavelengths and the delay ( $\sim 5$ days, Abdo et al. 2009) between the onset of $\gamma$-ray activity and the radio flare suggest that different emission regions are linked by the collimated jet. The $\gamma$-ray emission - related to inverse Compton (IC) scatterings - most likely occurs close to the compact object, while the radio emission - assumed to be synchrotron in origin - occurs farther out in the jet, at an angular distance from the core of a few tens of milli-arcseconds (e.g., Tudose et al. 2007, 2010), corresponding to $\sim 10^{15}-10^{16} \mathrm{~cm}$. The $\gamma$-ray modulation is due to the anisotropic efficiency of the IC scattering (Aharonian \& Atoyan 1981). Thus, the $\gamma$-ray maximum occurs at the superior conjuction (where the compact object is behind the WR star), when

\footnotetext{
$3 \gamma$-ray detections of Cygnus X-3 were reported in both the 1970s and 1980s at TeV (Vladimirsky et al. 1973; Danaher et al. 1981; Lamb et al. 1982) and PeV energies (Samorski \& Stamm 1983; Bhat et al. 1986). However, subsequent observations by more sensitive groundbased telescopes did not confirm $\mathrm{TeV}$ and $\mathrm{PeV}$ emission from this source (O'Flaherty et al. 1992). Furthermore, the COS-B satellite could not find any clear emission from Cygnus X-3 at MeV-GeV energies (Hermsen et al. 1987), and both CGRO/EGRET observations of the Cygnus region (1991-1994) and the first-year analysis of AGILE observations could not demonstrate that there was a solid association with the microquasar, although they confirmed a $\gamma$-ray detection above $100 \mathrm{MeV}$ in a region including Cygnus X-3 (Mori et al. 1997; Pittori et al. 2009).
}

relativistic electrons of the jet, moving towards the Earth, have head-on collisions with stellar UV photons. This orbital phase corresponds to the minimum of the X-ray modulation, produced in turn by the maximum of absorption/scattering by the companion's wind (Abdo et al. 2009; Dubus et al. 2010; Zdziarski et al. 2012a).

A hadronic scenario accounting for $\gamma$-ray emission in microquasars was discussed by Romero et al. (2003, 2005). Their model is based on the interaction of a mildly relativistic jet with the dense wind of the companion star, and the $\gamma$-ray emission is due to the decay of neutral pions $\left(\pi^{0}\right)$ produced by pp collisions.

Furthermore, $\mathrm{TeV}$ emission from relativistic jet in microquasars has been predicted by several models (e.g., see Atoyan \& Aharonian 1999). A search for very-high-energy (VHE) $\gamma$-rays from the microquasar GRS $1915+105$ with H.E.S.S. (High Energy Stereoscopic System) was carried out, but no significant detection was found in the direction of the source (H.E.S.S. Collaboration et al. 2009). On the other hand, hints of VHE $\gamma$-rays were found in Cygnus X-1 (Albert et al. 2007). The Major Atmospheric Gamma-ray Imaging Cherenkov Telescope (MAGIC) observed Cygnus X-3 several times between March 2006 and August 2009, during both its hard and soft states ${ }^{4}$, but no evidence of clear VHE $\gamma$-ray emission from the microquasar was found: an overall $2 \sigma$ upper limit to the integral flux was set at $2.2 \times 10^{-12}$ photons $\mathrm{cm}^{-2} \mathrm{~s}^{-1}$ for energies above $250 \mathrm{GeV}$ (Aleksić et al. 2010).

Here we present a comprehensive and homogeneous analysis of Cygnus X-3 that takes into account $\gamma$-ray events found in the data between 2007 November 2 and 2009 July 29, during the AGILE "pointing" mode data-taking. We analyzed a dataset previously published by Tavani et al. (2009a) and Bulgarelli et al. (2012a). We report here the whole AGILE-GRID monitoring of Cygnus X-3 during the "pointing" mode, to confirm that the $\gamma$-ray activity coincides with the same repetitive pattern of multiwavelength emission and to analyze in depth the overall $\gamma$-ray spectrum by assuming both leptonic and hadronic scenarios.

\section{Observations}

The AGILE scientific instrument (Tavani et al. 2009b) is very compact and characterized by two co-aligned imaging detectors operating in the energy ranges $30 \mathrm{MeV}-30 \mathrm{GeV}$ (GRID: Barbiellini et al. 2002; Prest et al. 2003) and 18-60 keV (SuperAGILE: Feroci et al. 2007), as well as by both an anticoincidence system (Perotti et al. 2006) and a calorimeter (Labanti et al. 2006). The performance of AGILE is characterized by large fields of view (2.5 and $1 \mathrm{sr}$ for the $\gamma$-ray and hard X-ray bands, respectively) and optimal angular resolution (PSF $=3.0^{\circ}$ at $100 \mathrm{MeV}$, and PSF $=1.5^{\circ}$ at $400 \mathrm{MeV}$; see Cattaneo et al. 2011).

Until mid-October 2009 AGILE had operated in "pointing" mode with fixed attitude; in November 2009, AGILE entered "scanning mode", which is characterized by a controlled rotation of the pointing axis.

During the "pointing" mode data-taking ( 2.5 years $)$, the AGILE satellite performed $\sim 100$ pointings with variable exposure times (of typically 3-30 days), drifting about 1 degree per day from the initial boresight direction to match the

\footnotetext{
4 The MAGIC telescope was also pointed at Cygnus X-3 after two $\gamma$-ray alerts from the AGILE-GRID team (the first one after the $\gamma$-ray event of 16-17 April 2008, and the second after the event of 13-14 July 2009, see Appendix A). In both cases, they found a $2 \sigma$ upper limit, for energies above $250 \mathrm{GeV}$, of $\sim 10^{-11}$ photons $\mathrm{cm}^{-2} \mathrm{~s}^{-1}$ (Aleksić et al. 2010).
} 
solar-panel illumination constraints ${ }^{5}$. In this configuration, the AGILE-GRID was characterized by enhanced performances in the monitoring capability of a given source, especially in the energy band 100-400 MeV (see Bulgarelli et al. 2012a, for details). Owing to the different pointing strategies of the AGILE and Fermi satellites, the high on-source cumulative exposure (between 100 and $400 \mathrm{MeV}$ ) of the AGILE-GRID may be fundamental in the observation of this particular source.

In this paper, we report an analysis based on the AGILEGRID data collected between 2007 November 2 and 2009 July 29 (the same dataset reported by Chen et al. 2011). During this period, AGILE repeatedly pointed at the Cygnus region for a total of $\sim 275$ days, corresponding to a net exposure time of $\sim 11 \mathrm{Ms}$. The detailed analysis of the dataset is presented in Appendix A. In this paper, we report seven $\gamma$-ray main events.

\section{The gamma-ray activity in a multiwavelength context}

\subsection{General characteristics of the gamma-ray events}

Figure 1 shows the comprehensive multiwavelength light curve of Cygnus X-3, to help us analyze the pattern of multi-frequency emission. The $\gamma$-ray activity detected by the AGILE-GRID is presented along with the hard X-ray fluxes from Swift $/ \mathrm{BAT}^{6}$ (15-50 keV), soft X-ray fluxes from RXTE/ASM ${ }^{7}(3-5 \mathrm{keV})$, and radio flux density (when available) from the AMI-LA ${ }^{8}$ $(15 \mathrm{GHz})$ and RATAN-600 ${ }^{9}(2.15,4.8,11.2 \mathrm{GHz})$ radio telescopes.

The aim of the cross-correlation between the AGILE-GRID light curve and the multiwavelength emission pattern is to discuss the $\gamma$-ray trigger criteria and compare them with those previously published by Tavani et al. (2009a), Bulgarelli et al. (2012a), and Corbel et al. (2012).

Observing the light curve in Fig. 1, as well as the detailed zooms in Fig. 2, we can note that

- there is a strong anticorrelation between the hard X-ray and $\gamma$-ray emission. Every local minimum of the hard X-ray light curve is associated with $\gamma$-ray emission detected by the AGILE-GRID (see also the weak $\gamma$-ray event detected on 2008 June 21, modified Julian date (MJD) = 54638.58 , in the plot of Fig. 1 where $\sqrt{T S}=2.77$, photon flux $=(131 \pm 61) \times 10^{-8}$ photons $\left.\mathrm{cm}^{-2} \mathrm{~s}^{-1}\right)$. Conversely, every time the AGILE-GRID detects $\gamma$-ray activity the system exhibits a deep local minimum of the hard X-ray light curve (Swift/BAT count rate $\lesssim 0.02$ counts $\mathrm{cm}^{-2} \mathrm{~s}^{-1}$ );

- every time we detect $\gamma$-ray activity, Cygnus X-3 is in a soft

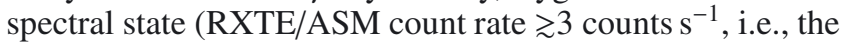
transitional level defined by Szostek et al. 2008);

- every time we detect $\gamma$-ray episodes (Table A.1 and red points in the AGILE-GRID light curve in Fig. 1), the system is moving towards either a major radio flare (radio flux

\footnotetext{
5 A detailed schedule of the AGILE observations - with the pointing starting coordinates and observation starting and ending times - is available online at http://agile.asdc.asi.it/current_pointing. html

6 Swift/BAT (Burst Alert Telescope) transient monitor results provided by the Swift/BAT team.

7 Rossi X-ray Timing Explorer (RXTE), All-Sky Monitor (ASM). Quick-look results provided by the RXTE/ASM team.

8 Courtesy of the Arcminute Microkelvin Imager (AMI) team.

9 Courtesy of Trushkin and the RATAN-600 team.
}

density $\gtrsim 1 \mathrm{Jy}$ ) or a quenched state preceding a major radio flare.

In Table 1, we report a brief synthesis of the multi-frequency pattern of emission of each main $\gamma$-ray event $(\sqrt{T S} \geqslant 3)$ detected by the AGILE-GRID. The average delay between the $\gamma$-ray event and the subsequent radio flare is $\overline{\Delta T_{2}} \approx 4$ days, which is consistent with the value ( $5 \pm 7$ days) found by Abdo et al. (2009).

If we refer to the third column in Table 1 , we can see that the transient $\gamma$-ray emission occurs when the system is either moving into a quenched state ("pre-quenched") or towards a radio flare ("pre-flare"), which has always been observed after a quenched state, i.e., the $\gamma$-ray emission is detected when the system is moving into or out of a quenched state. Hence, from a purely phenomenological point of view, the quenched state seems to be a "key" condition for the $\gamma$-ray emission.

We stress in general that $\gamma$-ray events - always during soft states - occur in the proximity of spectral X-ray transitions. In particular, we found that intense transient $\gamma$-ray emission is detected both immediately after hard-to-soft (e.g., the events of 2-3 November 2008 [MJD = 54773.17] and 20-21 June 2009 $[\mathrm{MJD}=55003.37]$ ) and before soft-to-hard spectral X-ray transitions (e.g., the events of 16-17 April 2008 [MJD = 54 573.08], 11-12 December 2008 [MJD = 54812.39], and 13-14 July 2009 [MJD = 55 025.55]). Observing Fig. 1 and the zooms in Fig. 2, we can note that strong transient $\gamma$-ray emission generally occurs when the system has just entered into or is moving out of a prominent minimum of the Swift/BAT light curve (e.g., November-December 2008 [MJD $\simeq 54770-54815$ ] and June-July 2009 [MJD $\simeq 55$ 000-55040] events).

This comprehensive study confirms that the $\gamma$-ray emission conditions of Cygnus X-3, during the whole "pointing" monitoring by the AGILE satellite, agree completely with the ones found by Tavani et al. (2009a), Bulgarelli et al. (2012a), and Corbel et al. (2012).

\subsection{Peculiarities of the gamma-ray events}

\subsubsection{The gamma-ray event of 11-12 February 2008 $(\mathrm{MJD}=54507.19)$}

A more detailed discussion is needed for the $\gamma$-ray event of 11-12 February 2008 (MJD $=54507.19$, see the upper-left panel in Fig. 2), which is a special event among the AGILEGRID detections.

The $\gamma$-ray activity in this period occurred when Cygnus X-3 was at the transitional level between its soft and hard X-ray

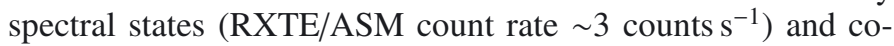
incides with a short but sharp dip in the Swift/BAT light curve at MJD $=54508$, count rate $=(0.019 \pm 0.005)$ counts cm $\mathrm{cm}^{-2} \mathrm{~s}^{-1}$, DATA_FLAG $=0$ (data quality flag $=$ good $)$. Even if the event does not coincide with a bright soft state, it seems to confirm the simultaneous $\gamma$-ray-event/hard-X-ray-minimum occurrences that we find in all other cases. There are no available radio data for this period.

\subsubsection{The gamma-ray event of 20-21 June 2009 $(\mathrm{MJD}=55003.37)$}

The $\gamma$-ray event on 20-21 June 2009 (MJD = 55 003.37) occurred at the beginning of a quenched radio state (see the lowerright panel in Fig. 2). Unfortunately, we have no radio data covering the subsequent days (from the third to the ninth day after the $\gamma$-ray event), but we cannot exclude the presence of a 


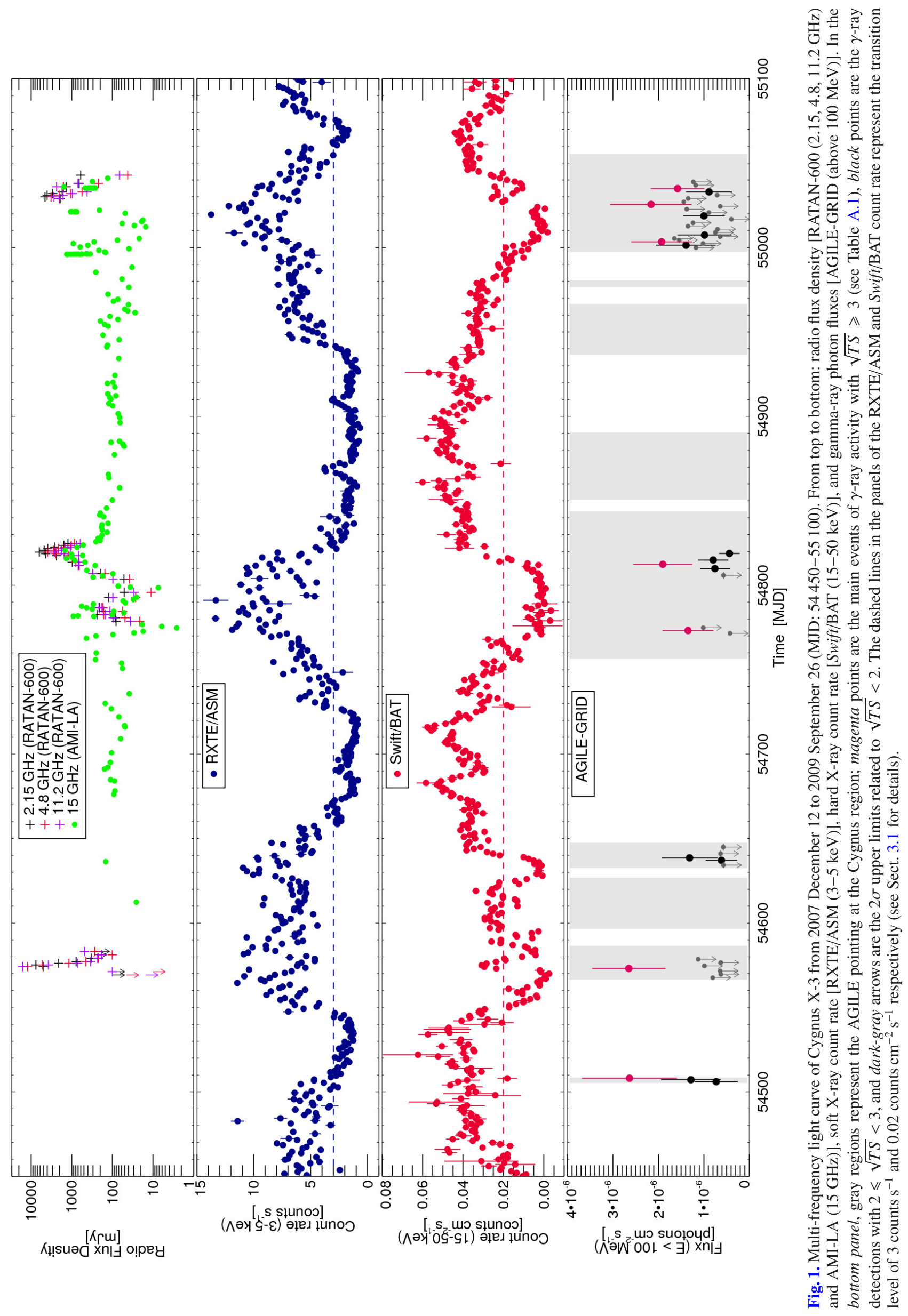



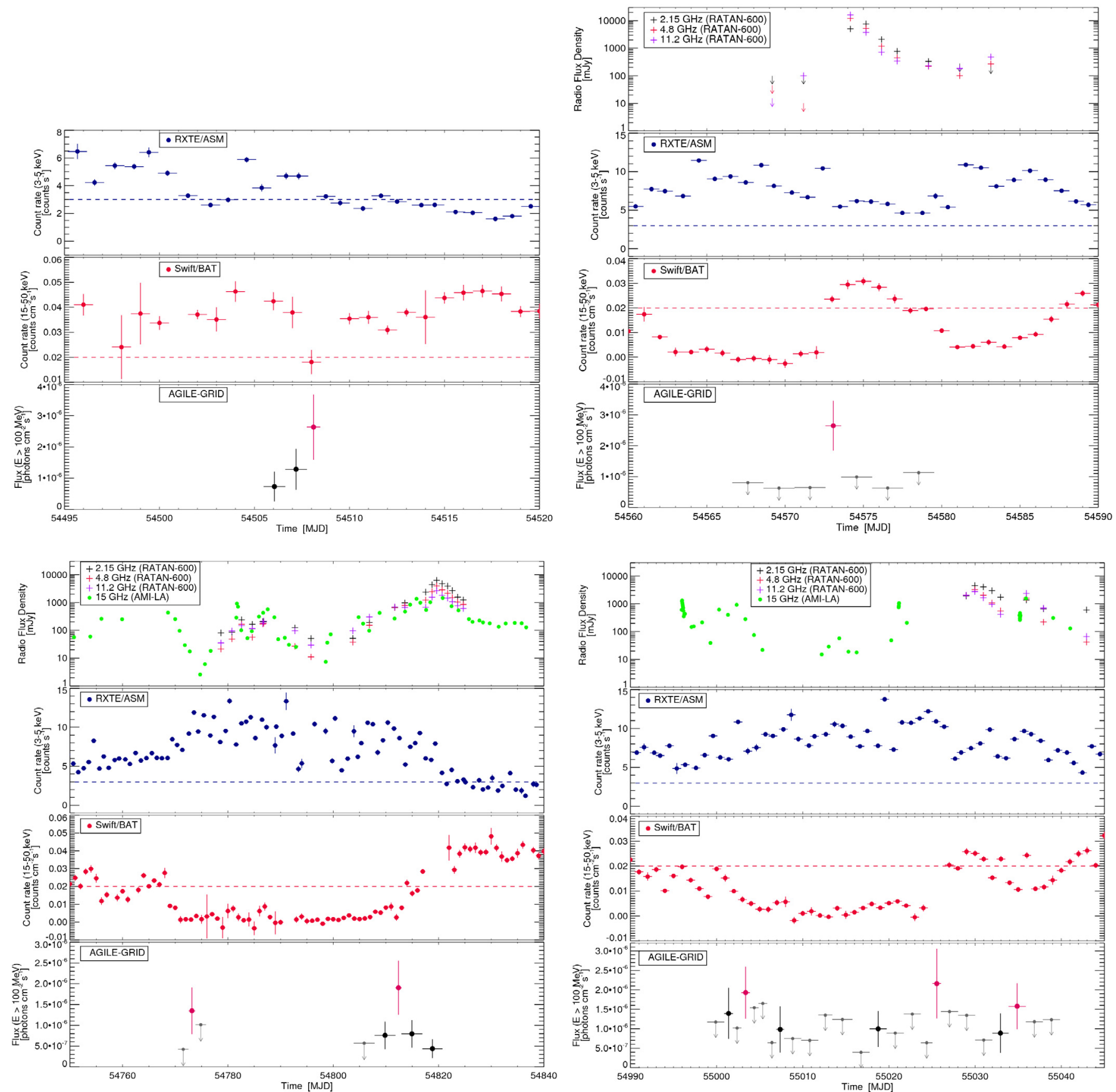

Fig. 2. Multi-frequency light curves centered on the main events of $\gamma$-ray activity detected by the AGILE-GRID (detailed views of the main plot in Fig. 1). Upper-left plot: from 2008 January 30 to 2008 February 24 (MJD: 54 495-54 520). Upper-right plot: from 2008 April 4 to 2008 May 4 (MJD: 54 560-54 590). Lower-left plot: from 2008 October 11 to 2009 January 9 (MJD: 54 750-54 840). Lower-right plot: from 2009 June 8 to 2009 August 2 (MJD: 54 990-55 045).

major radio flare - soon after the quenched state - that might have escaped detection. In Fig. 2 (lower-right panel), we note that the AMI-LA light curve $(15 \mathrm{GHz})$ shows a subsequent radio flare $\sim 18$ days after the $\gamma$-ray event ( $\sim 13$ days after the quenched state), possibly corresponding to a delayed radio burst $(\mathrm{MJD}=55021.2$, radio flux density $=1.06 \mathrm{Jy})$.

\subsubsection{The long-term gamma-ray emission June-July 2009 $(M J D \simeq 55000-55040)$}

Finally, we note that the extended $\gamma$-ray activity of June-July 2009 (see the lower-right panel in Fig. 2), occurred during intense radio activity (with a high average radio flux density) coincident with a long-lasting soft X-ray spectral state. It is important to remark that this period coincides with one of the two temporal windows of strong $\gamma$-ray activity detected by Fermi-LAT, with a peak photon flux greater than $\sim 200 \times 10^{-8}$ photons $\mathrm{cm}^{-2} \mathrm{~s}^{-1}$ (Abdo et al. 2009). This $\gamma$-ray peak is simultaneous (and consistent) with the event detected by the AGILE-GRID on 21-23 July 2009 (photon flux $=(158 \pm 59) \times 10^{-8}$ photons $\left.\mathrm{cm}^{-2} \mathrm{~s}^{-1}\right)$. These data (June-July 2009) are discussed in more detail in a dedicated paper on the $\gamma$-ray emission of the microquasar during the mid-2009/mid-2010 period (Bulgarelli et al. 2012a). 
Table 1. Main events of $\gamma$-ray activity $(\sqrt{T S} \geqslant 3)$ detected by the AGILE-GRID.

\begin{tabular}{lcccc}
\hline \hline MJD & $\begin{array}{c}\text { X-ray } \\
\text { state }\end{array}$ & $\begin{array}{c}\text { Radio } \\
\text { state }\end{array}$ & $\begin{array}{c}\Delta T_{2} \\
{[\text { days }]}\end{array}$ & $\begin{array}{c}\text { Radio } \\
\text { flux density }\end{array}$ \\
\hline 54507.19 & $\begin{array}{l}\text { X-ray state } \\
\text { trans. level }\end{array}$ & $(?)$ & $(?)$ & $(?)$ \\
54573.08 & Soft & Pre-flare & $\sim 1$ & $\approx 16 \mathrm{Jy}(11.2 \mathrm{GHz})$ \\
54773.17 & Soft & Pre-quenched & $\sim 8$ & $\approx 1 \mathrm{Jy}(15 \mathrm{GHz})$ \\
54812.39 & Soft & Pre-flare & $\sim 6$ & $\approx 3 \mathrm{Jy}(11.2 \mathrm{GHz})$ \\
55003.37 & Soft & Pre-quenched & $(?)$ & $(?)$ \\
55025.55 & Soft & Pre-flare & $\sim 3$ & $\approx 3 \mathrm{Jy}(11.2 \mathrm{GHz})$ \\
55034.88 & Soft & Pre-flare & $\sim 1$ & $\approx 2.3 \mathrm{Jy}(11.2 \mathrm{GHz})$ \\
& & & & $\approx 1.6 \mathrm{Jy}(15 \mathrm{GHz})$ \\
\hline
\end{tabular}

Notes. Column 1: date of the $\gamma$-ray event (average in MJD). Column 2: $\mathrm{X}$-ray spectral state. Column 3: radio-flux-density state at the time of the $\gamma$-ray activity. Column 4: time delay $\left(\Delta T_{2}\right)$ in days between the $\gamma$-ray event and the major radio flare. Column 5: radio flux density of the major radio flare.

\section{Modeling the spectral energy distribution}

By accounting for the X-ray, $\gamma$-ray (AGILE-GRID), and TeV emission (MAGIC spectral upper limits), we modeled the multiwavelength spectral energy distribution (SED) of Cygnus X-3 during a soft spectral state, with both the leptonic and hadronic scenarios. We considered an X-ray spectrum measured by RXTE-PCA ${ }^{10}$ and RXTE-HEXTE ${ }^{11}(\sim 3-150 \mathrm{keV})$ when the source was in a "hypersoft" state (Koljonen et al. 2010), the AGILE-GRID spectrum for the main $\gamma$-ray events (Fig. A.1), and the MAGIC differential flux upper limits obtained when the source was in the soft state. (Aleksić et al. 2010). The hypersoft state of Cygnus X-3, which is a subclass of the ultrasoft state defined in Hjalmarsdotter et al. (2009), is usually exhibited by the microquasar during the quenching/pre-flaring radio activity $^{12}$. This X-ray spectral state is characterized by a weak and hard power-law tail ( $\alpha=1.7-1.9)$ of non-thermal origin.

Thus, we analyzed a pattern of multiwavelength datasets that, even if not acquired simultaneously, are qualitatively consistent because they all refer to the same spectral state of Cygnus X-3: the X-ray and AGILE-GRID datasets are related to the soft-state activity preceding the radio major flares and the $\mathrm{TeV}$ data are related to the soft-state activity following the radio major flares (MAGIC has never observed Cygnus X-3 during its pre-flaring radio states).

\subsection{A leptonic scenario}

We modeled the multi-frequency SED by assuming a simple leptonic scenario in which a plasmoid of high energy electrons/positrons, injected into the jet structure, upscatters via inverse Compton interactions soft seed photons from both the WR star and the accretion disk.

\footnotetext{
${ }^{10}$ Proportional Counter Array (PCA).

${ }^{11}$ High Energy X-ray Timing Experiment (HEXTE).

${ }^{12}$ This is an average "hypersoft" spectrum related to 28 pointed RXTE observation between February 2000 and January 2006 (see the supporting information of Koljonen et al. 2010, for details).
}

Our aim is to analyze a possible link between the power-law tail of this special soft X-ray spectral state and the $\gamma$-ray emission detected by the AGILE-GRID.

The physical parameters of the photon field are literaturebased. We modeled the X-ray data with a black body (BB) spectrum $^{13}$ characterized by a temperature $T_{\mathrm{bb}} \approx 1.3 \mathrm{keV}$, which is consistent with the typical characteristic temperature of the disk during the hypersoft/ultrasoft state (Hjalmarsdotter et al. 2009; Koljonen et al. 2010), and a $L_{\mathrm{bb}} \approx 8 \times 10^{37} \mathrm{erg} \mathrm{s}^{-1}$. The main parameters that we used for the WR star are $T_{\star}=$ $10^{5} \mathrm{~K}$ and $L_{\star} \approx 10^{39} \mathrm{erg} \mathrm{s}^{-1}$ (see Dubus et al. 2010). The WR star is assumed to emit UV photons isotropically. We modeled the average $\gamma$-ray emission in the orbital phase. Thus, the WR photons are assumed to come mainly from the side of the jet and collide with the relativistic leptons via IC scattering processes.

We carried out two different models: in the first one (leptonic model " $A$ "), the plasmoid interacts with the soft photon bath "close" to the disk (the star-plasmoid distance is $R \approx d \approx$ $3 \times 10^{11} \mathrm{~cm}$ ), whereas in the second one (leptonic model " $B$ ”) the interaction region is "far away" from the accretion disk (the star-plasmoid distance is $R \approx 10 d \approx 3 \times 10^{12} \mathrm{~cm}$ ).

For both models, the inclination of the jet to the line of sight is assumed to be $i=14^{\circ}$, and the plasmoid is assumed to be spherical (radius $r=3 \times 10^{10} \mathrm{~cm}$ ) with a bulk motion characterized by a Lorentz factor of $\Gamma=1.5(v=\sqrt{5} c / 3)$. The population of electrons is modeled by a broken-power-law spectral distribution, with spectral indices $\alpha_{1}=2.2, \alpha_{2}=4.0, \gamma_{\min }=1$, $\gamma_{\max }=10^{5}$, and an energy break of $\gamma_{\mathrm{b}}=4 \times 10^{3}$

$$
\frac{\mathrm{d} N}{\mathrm{~d} \gamma \mathrm{d} V}=\frac{K_{\mathrm{e}} \gamma_{\mathrm{b}}^{-1}}{\left(\frac{\gamma}{\gamma_{\mathrm{b}}}\right)^{\alpha_{1}}+\left(\frac{\gamma}{\gamma_{\mathrm{b}}}\right)^{\alpha_{2}}} \quad\left[\gamma_{\min } \leqslant \gamma \leqslant \gamma_{\max }\right]
$$

The spectral indices and the energy break of the electron distribution are the best-fit values for the AGILE-GRID spectral shape. The distribution of electrons/positrons is assumed to be isotropic in the plasmoid rest frame (the jet comoving frame). We adopted the Klein-Nishina formula to describe the Compton scattering of soft photons by a cloud of mildly relativistic leptons (Aharonian \& Atoyan 1981).

In the leptonic model " $A$ ", the distance from the star to the plasmoid location is assumed to be $R \approx 3 \times 10^{11} \mathrm{~cm}(R \approx d)$, i.e., the plasmoid in the jet is very close both to the compact object and the accretion disk. The distance between the plasmoid center and the compact object is $H \approx 3 \times 10^{10} \mathrm{~cm}$, i.e., $H \approx r$. The results of this modeling are presented in Fig. 3 . The electron number density of the plasmoid is $n_{\mathrm{e}} \approx 3 \times 10^{9}$ electrons $\mathrm{cm}^{-3}$ (the prefactor in Eq. (1) is $K_{\mathrm{e}}=2 \times 10^{5} \mathrm{~cm}^{-3}$, and the integrated number of electrons is $\left.N_{\mathrm{e}}=3 \times 10^{41}\right)$. We took into account the $\gamma \gamma$ absorption (for $\mathrm{e}^{ \pm}$pair production) of the IC $\gamma$-ray photons by the X-ray photons from the accretion disk. We assumed that the distribution of the disk photons is fully isotropized by the stellar wind in the observer frame. This implies that the $\gamma$-ray photosphere (i.e., where $\tau_{\gamma \gamma} \geqslant 1$ ) has a radius of $\sim 10^{10} \mathrm{~cm}$ (Cerutti et al. 2011). With these assumptions,

\footnotetext{
13 Our modeling is a simplification: we assume that the bump in the X-ray emission during the "hypersoft" state can be modeled with a simple BB component, which is a very good approximation for our purposes. In this state, the overall X-ray emission is totally dominated by strong BB emission from the accretion disk. Nevertheless, more accurate modeling should be based on a Comptonized $\mathrm{BB}$ spectrum of the corona (see Koljonen et al. 2010, for details).
} 


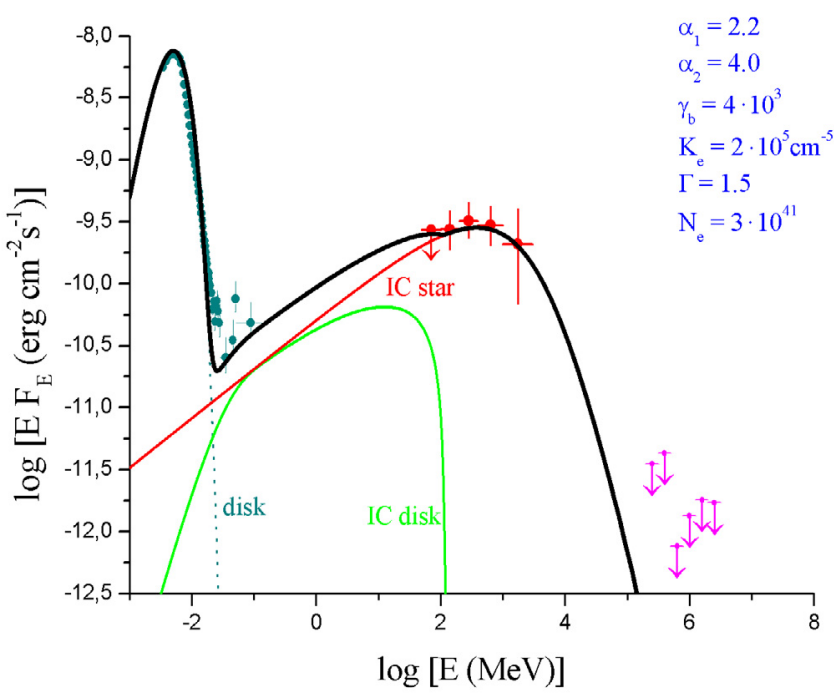

Fig. 3. Multiwavelength SED of Cygnus X-3 during the main $\gamma$-ray events (non-simultaneous data) and the leptonic model " $A$ " (see main text). Blue circles: X-ray average "hypersoft" spectrum (Koljonen et al. 2010), RXTE-PCA and RXTE-HEXTE data ( 3 to $\sim 150 \mathrm{keV})$; red circles: AGILE-GRID energy spectrum (50 MeV to $3 \mathrm{GeV}$ ) of the main $\gamma$-ray episodes (Figs. A.1 and A.2); magenta arrows: MAGIC differential flux upper limits (95\% C.L.), $199-3155 \mathrm{GeV}$, related to soft spectral state (Aleksić et al. 2010). Spectral components of the model are the BB emission from the disk (blue short-dashed line), IC scattering of the soft photons from the accretion disk (green solid line), and IC scattering of the soft stellar photons (red solid line). The global SED model curve is indicated by a black solid line.

the lowest part of the plasmoid is within the $\gamma$-ray photosphere ${ }^{14}$. The spectral component related to the IC scatterings of the disk photons (green curve) is actually produced in this region, very close to the disk, where the X-ray photon density as well as the optical depth is high. Since $\tau_{\gamma \gamma}>1$, this component displays a sharp cut-off energy at $\sim 100 \mathrm{MeV}$ (i.e., the threshold for $\mathrm{e}^{ \pm}$production, given the characteristic energies of the disk photons). On the other hand, the spectral component related to the IC scatterings of the stellar wind photons (red curve) does not show any cut-off energy, because it is mainly produced in the farthest part of the plasmoid (outside the $\gamma$-ray photosphere, for distances greater than $\sim 10^{10} \mathrm{~cm}$ from the disk), where the $\gamma \gamma$ absorption by the X-ray disk photons is negligible. Thus, we deduced that in our geometry the plasmoid volume outside the $\gamma$-ray photosphere emits the bulk of the $\gamma$-ray emission above $100 \mathrm{MeV}$ via IC processes acting on stellar photons (see Fig. 3).

In model " $A$ ", assuming a lepton injection rate of $\dot{N}_{\mathrm{e}}=$ $n_{\mathrm{e}} \pi r^{2} v \approx 2 \times 10^{41}$ leptons $\mathrm{s}^{-1}$, the jet kinetic luminosity for the leptons $\left(L_{\text {kin, e }}=\dot{N}_{\mathrm{e}} \Gamma m_{\mathrm{e}} c^{2}\right)$ would be $L_{\text {kin, e }}^{A} \approx 2 \times 10^{35} \mathrm{erg} \mathrm{s}^{-1}$.

In the leptonic model " $B$ ", the distance from the star to the plasmoid is assumed to be $R \approx 3 \times 10^{12} \mathrm{~cm}(R \approx 10 d)$, i.e., the plasmoid in the jet is far away from the compact object and the accretion disk. The distance between the plasmoid center and the compact object is $H \approx 3 \times 10^{12} \mathrm{~cm}$, i.e., $R \approx H$. We assumed that the disk photons enter the plasmoid mainly from behind. The results of this modeling are shown in Fig. 4. The electron density of the plasmoid is $n_{\mathrm{e}} \approx 1.5 \times 10^{11}$ electrons $\mathrm{cm}^{-3}$, where the prefactor in Eq. (1) is $K_{\mathrm{e}}=8 \times 10^{6} \mathrm{~cm}^{-3}$ and the integrated number of electrons is $N_{\mathrm{e}}=1.5 \times 10^{43}$. In

\footnotetext{
${ }^{14}$ Assuming that the photosphere radius is equal to the plasmoid radius, i.e., $3 \times 10^{10} \mathrm{~cm}$, the fraction of the plasmoid volume inside the $\gamma$-ray photosphere is $\sim 32 \%$.
}

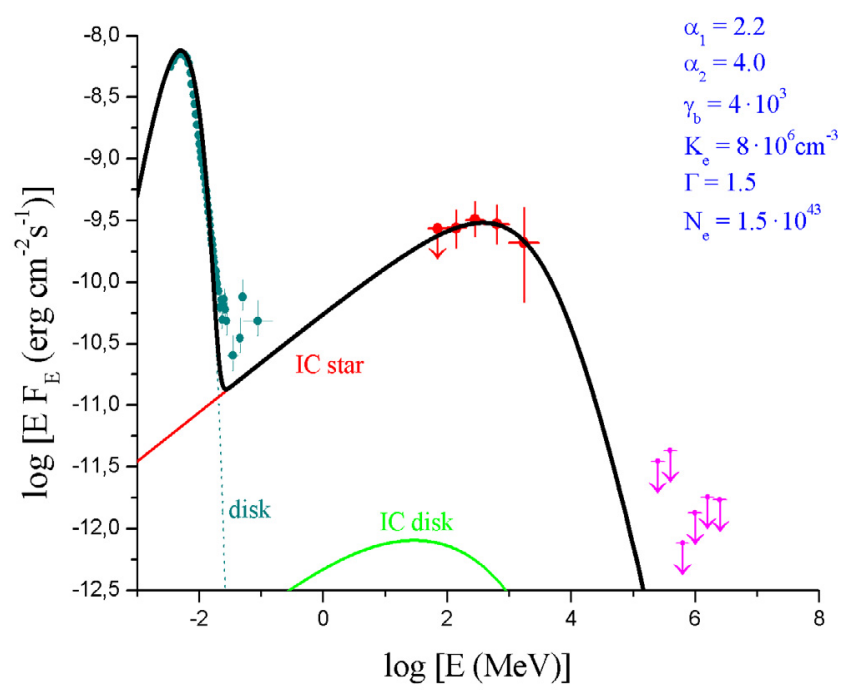

Fig. 4. Multiwavelength SED of Cygnus X-3 during the main $\gamma$-ray events (non-simultaneous data) and the leptonic model " $B$ " (see main text). Spectral components of the model are the BB emission from the disk (blue short-dashed line), IC scattering of the soft photons from the accretion disk (green solid line), and IC scattering of the soft stellar photons (red solid line). The global SED model curve is indicated by a black solid line. For a detailed description of the datasets, see caption to Fig. 3.

this model, the spectral component related to the IC scatterings of disk photons (green curve) is negligible compared to the IC component of soft photons from the star (red curve). We note that the "IC disk" component does not show any cut-off energy related to the $\gamma \gamma$ absorption by X-ray photons, because the IC $\gamma$-rays are produced well outside the $\gamma$-ray photosphere (at distances $\gg 10^{10} \mathrm{~cm}$ ).

In model " $B$ ", assuming a lepton injection rate of $\dot{N}_{\mathrm{e}}=$ $n_{\mathrm{e}} \pi r^{2} v \approx 10^{43}$ leptons $\mathrm{s}^{-1}$, the jet kinetic luminosity for the leptons $\left(L_{\mathrm{kin}, \mathrm{e}}=\dot{N}_{\mathrm{e}} \Gamma m_{\mathrm{e}} c^{2}\right)$ would be $L_{\text {kin, e }}^{B} \approx 10^{37} \mathrm{erg} \mathrm{s}^{-1}$.

In these models, the expected VHE $\gamma$-ray emission would be very faint. These expectations are consistent with the MAGIC upper limits, and might explain the lack of $\mathrm{TeV}$ bright detections during soft states.

\subsection{A hadronic scenario}

We also considered a "hadronic scenario" for $\gamma$-ray production from Cygnus X-3. In our model, we used the same formalism adopted by Romero et al. (2003). In this case, the compact source is assumed to eject a flux of mildly relativistic hadrons (mostly protons) at the base of the jet. These protons are first accelerated near the compact object and then propagate along the jet interacting with the gaseous surroundings provided by the WR companion mass-outflow. The resulting proton-proton ( $\mathrm{pp}$ ) collisions can copiously produce pions and $\gamma$-rays resulting from neutral pion decays.

The proton distribution in the jet is assumed to be isotropic in the jet comoving frame, with an energy spectrum described by a power law with a high energy cut-off

$$
\frac{\mathrm{d} N}{\mathrm{~d} \gamma \mathrm{d} V}=K_{\mathrm{p}} \gamma^{-\alpha} \exp \left(-\gamma / \gamma_{\mathrm{c}}\right) \quad\left[\gamma \geqslant \gamma_{\min }\right]
$$

with $\alpha=3, \gamma_{\min }=1$, and $\gamma_{\mathrm{c}}=100$. The spectral index of the distribution is the best-fit value for the AGILE-GRID spectral shape. We set the energy cut-off value at $\gamma_{\mathrm{c}}=100$ so 


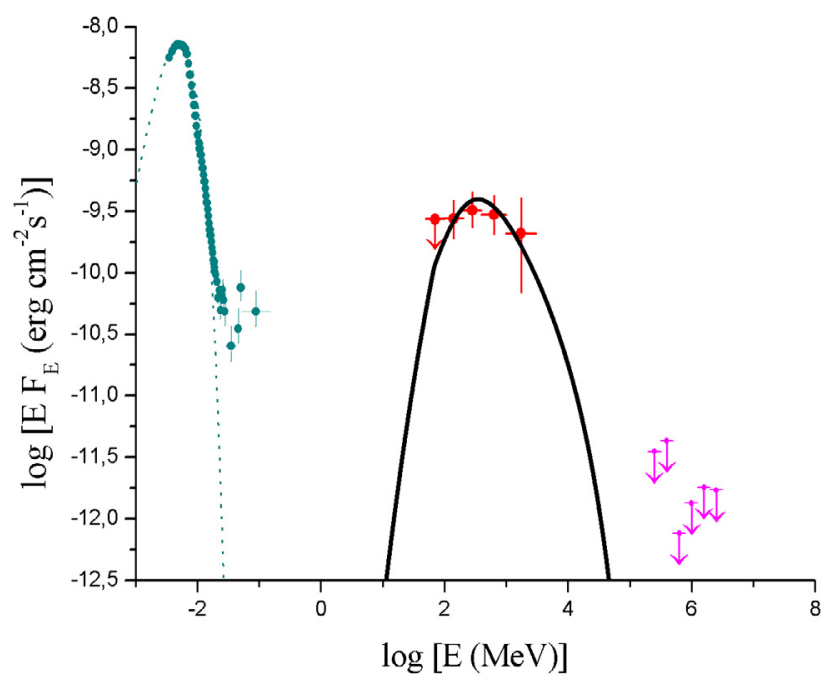

Fig. 5. Multiwavelength SED of Cygnus X-3 during $\gamma$-ray events (nonsimultaneous data) and a hadronic model (see main text). Black body emission from the disk (blue short-dashed line), and $\gamma$-ray emission from $\pi^{0}$-decays (black solid line) are plotted. For a detailed description of the datasets, see caption to Fig. 3 .

that the total SED is consistent with the spectral constraints of the MAGIC upper limits.

The ejected protons interact with the hadronic matter of the WR strong wind. The inelastic hadronic scatterings produce neutral pions that subsequently decay into $\gamma$-rays. We adopted the same formula for the cross section $\sigma_{\mathrm{pp}}(\gamma)$ of inelastic pp interaction reported by Kelner et al. (2006). We assumed that the injected protons in the jet interact with the gas of the wind along a cylindrical column of matter ${ }^{15}$ with a radius $r=3 \times 10^{10} \mathrm{~cm}$ and a height of $H \approx 3 \times 10^{12} \mathrm{~cm}$ (this height provides the interesting part of the cylinder in which most of the interactions take place). In analogy with the leptonic models, we assumed for the jet a bulk Lorentz factor of $\Gamma=1.5$, and an inclination to the line of sight of $i=14^{\circ}$. To quantify the density of matter in the WR wind, we assumed that the companion star has a mass-loss rate of $\dot{M} \sim 10^{-5} M_{\odot} \mathrm{yr}^{-1}$ and the speed of the wind is $v_{\text {wind }} \sim 1000 \mathrm{~km} \mathrm{~s}^{-1}$ (Szostek \& Zdziarski 2008). By integrating the density of matter in this cylinder expressed in terms of the number density of protons $\left(\varrho \sim 1 / R^{2}\right.$, where $R$ is the distance from the star), we find that the total number of protons from the wind in this column is $N_{\mathrm{p} \text {, wind }} \approx 3.7 \times 10^{45}$.

We considered a variety of proton injection rates in the jet, $\dot{N}_{\mathrm{p}, \text { jet }}$. The result of our best-fit hadronic model for Cygnus X-3 is reported in Fig. 5. In this case, the integrated number of protons injected in the jet is $N_{\mathrm{p}, \mathrm{jet}} \approx 9.0 \times 10^{42}$, and the average proton number density in the column of interest is $n_{\mathrm{p}, \mathrm{jet}} \approx 1.1 \times 10^{9}$ protons $\mathrm{cm}^{-3}$. The proton injection flux in the jet of our best-fit model turns out to be $\phi_{\mathrm{p}, \mathrm{jet}} \approx 2.4 \times 10^{19}$ protons $\mathrm{cm}^{-2} \mathrm{~s}^{-1}$, which corresponds to a proton injection rate of $\dot{N}_{\text {p,jet }} \approx 6.7 \times 10^{40}$ protons s$^{-1}$.

In Cygnus X-3, the corresponding jet kinetic luminosity for the hadrons $\left(L_{\mathrm{kin}, \mathrm{p}}=\dot{N}_{\mathrm{p}} \Gamma m_{\mathrm{p}} c^{2}\right)$ would be $L_{\mathrm{kin}, \mathrm{p}} \approx$ $1.5 \times 10^{38} \mathrm{erg} \mathrm{s}^{-1}$. This value is consistent with the average bolometric luminosity of the hypersoft state, $L_{\mathrm{bol}}^{\mathrm{HYS}} \approx 1.2 \times$ $10^{38} \mathrm{erg} \mathrm{s}^{-1}$ (Koljonen et al. 2010). Moreover, $L_{\text {kin, p }}$ is lower than the Eddington accretion limit for the system, which is

\footnotetext{
15 A cylindrical configuration of the jet corresponds to setting $\epsilon=0$ in the formalism adopted by Romero et al. (2003) to describe the jet radius dependence on the axis, $r(z)=\xi z^{\epsilon}$.
}

$L_{\text {Edd }} \approx 10^{39} \mathrm{erg} \mathrm{s}^{-1}$ assuming that the compact object is a black hole with a mass of $M_{x} \approx 10 M_{\odot}$.

\section{Discussion}

In the context of a leptonic scenario, we find that most of the $\gamma$-ray emission above $100 \mathrm{MeV}$ is due to IC scatterings of stellar wind photons by relativistic electrons (see Figs. 3 and 4), according to the results of Dubus et al. (2010) and Zdziarski et al. (2012a). We note that the IC cooling times are very short $\left(t_{\mathrm{IC}} \sim 1-10 \mathrm{~s}\right)$. Thus, the observed time-scale of $\gamma$-ray emission (1-2 days) and the strong orbital modulation ( $\sim 4.8 \mathrm{~h})$ detected in $\gamma$-rays (Abdo et al. 2009) impose a continuous injection of accelerated particles into the jet. The $\gamma$-ray emission, as also noted by Zdziarski et al. (2012a), cannot be related to a single injection of a relativistic plasmoid in the jet. Our leptonic picture may suggest that there is a substructure. We find that the innermost part of the jet, where the density of X-ray disk photons is relatively high, could contribute significantly to the hard X-rays at $\sim 100 \mathrm{keV}$. In model " $A$ ", the spectral component related to IC scatterings of soft photons from the disk (green curve) gives a substantial contribution to the overall model below $\sim 100 \mathrm{MeV}$ that is consistent with the hard tail of the X-ray dataset (see Fig. 3). This model suggests that there is a possible spectral link between the power-law tail of ultrasoft/hypersoft state and the $\gamma$-ray emission detected by the AGILE-GRID. On the other hand, in the region of the jet outside the $\gamma$-ray photosphere related to pair production on disk photons $\left(H \gtrsim 10^{10} \mathrm{~cm}\right)$, the "IC disk" component becomes very low at $\sim 100 \mathrm{keV}$ and the overall contribution to the hard X-rays is negligible (Fig. 4). Thus, according to model " $B$ ", if the region of $\gamma$-ray emission is far away from the compact object, the power-law tail in the hard X-ray band cannot be accounted for by IC processes in the jet. For this reason, we assumed two "extreme" cases in the spatial configuration of the $\gamma$-ray emitting blob, which is located at $H \approx 3 \times 10^{10} \mathrm{~cm}$ (model " $A$ ") and $H \approx 3 \times 10^{12} \mathrm{~cm}$ (model " $B$ "). Dubus et al. (2010) found that the $\gamma$-ray emitting region is located at a distance $H \lesssim 10 d \approx 3 \times 10^{12} \mathrm{~cm}$. By assuming that the compact object is a black hole with a mass of $M_{x}=20 M_{\odot}$, their simulation found that the best fit to the $\gamma$-ray modulation was for $H \approx 3 \times 10^{11} \mathrm{~cm}$. Hence, the bulk of the $\gamma$-ray emission is possibly produced at an intermediate configuration between models " $A$ " and " $B$ ".

The leptonic picture " $B$ " is qualitatively consistent with the one proposed by Zdziarski et al. (2012a) to explain the Fermi-LAT data published in Abdo et al. (2009), even if the peak energy of the IC bump is quite different: in our models the peak energy is between $\sim 100 \mathrm{MeV}$ and $\sim 1 \mathrm{GeV}$, whereas in their models the peak energy is between $\sim 3 \mathrm{MeV}$ and $\sim 50 \mathrm{MeV}$. This difference could be due to the unequal spectral indices of the $\gamma$-ray spectra detected by the AGILE-GRID and FermiLAT (see Fig. A.2). Moreover, Zdziarski et al. (2012a) assumed that the electrons are injected in the jet with a power-law rate for $\gamma_{1} \leqslant \gamma \leqslant \gamma_{2}$. The electrons subsequently lose energy via Compton, synchrotron, and adiabatic losses and form a distribution below $\gamma_{1}$. Thus, they demonstrated that models with $\gamma_{1}<$ $10^{3}$ contribute significantly to the hard X-rays, which appears to conflict with the observed orbital modulation at $\sim 100 \mathrm{kev}$ during the $\gamma$-ray emitting intervals, which is out of phase with the $\gamma$-ray modulation at $>100 \mathrm{MeV}$ (Zdziarski et al. 2012b). This phase misalignment would rule out any substantial contribution of the jet to the hard X-rays, in (apparent) contradiction with our findings for model " $A$ ". We first remark that in our models the energy distributions of the accelerated particles 
are assumed to be "steady-state" spectra, arising from physical cooling processes, and not injected spectra. Our leptonic models are then based on the IC scatterings of both UV stellar photons and X-ray disk photons, whereas Zdziarski et al. (2012a) consider the UV stellar photons only (neglecting the X-ray photons from the accretion disk). By observing Fig. 3, we note that at $\sim 100 \mathrm{keV}$ the contribution of the "IC disk" component (green curve) is equivalent to the contribution of the "IC star" component (red curve). However, the latter has a modulated emission (the jet-wind geometry is anisotropic during the orbital phase), whereas the former is unmodulated (the jet-disk geometry does not significantly change with orbital phase). Thus, the effective unabsorbed modulation at $\sim 100 \mathrm{keV}$ - that is a superposition of a modulated and a unmodulated component - should have a lower amplitude than the effective modulation at energies $>100 \mathrm{MeV}$, which is actually related only to the modulated "IC star" component. Moreover, anisotropic absorption effects in the wind could strongly affect the unabsorbed $100 \mathrm{keV}$ emission and produce an observed modulation in phase with the soft X-ray band.

Finally, we note that, for energies higher than $\sim 10 \mathrm{GeV}$, $\gamma$-rays are above the threshold for pair production on stellar photons. Nevertheless, Zdziarski et al. (2012a) demonstrated that, for a similar choice of geometrical parameters, the value of the optical depth is moderate and peaks at $\sim 0.1-1 \mathrm{TeV}$. Thus, the $\gamma \gamma$ absorption by UV stellar photons was neglected in our (leptonic and hadronic) models.

In the context of a hadronic scenario, we used a model similar to the one proposed by Romero et al. (2003). The only substantial difference consists in the jet geometry: we used a cylindrical model, whereas they used a conical configuration. We found that a simply hadronic model can account for the $\gamma$-ray spectrum detected by the AGILE-GRID, by assuming a reasonable proton injection rate in the jet. It is interesting to compare our best-fit hadronic injection rate for Cygnus X-3 with the value deduced for the microquasar SS433, which is known to produce a quasi-steady jet of hadronic nature (Migliari et al. 2002). SS443 is characterized by jet mass-ejection rates near $\dot{M}_{\text {jet }} \approx 5 \times 10^{-7} M_{\odot} \mathrm{yr}^{-1}$ (Konigl 1983; Fabrika \& Borisov 1987; Reynoso et al. 2008), which corresponds to a proton injection rate of $\dot{N}_{\mathrm{p} \text {,jet }}^{\mathrm{SS} 443} \approx 1.9 \times 10^{43}$ protons s$^{-1}$. Thus, we have that $\dot{N}_{\mathrm{p}, \mathrm{jet}}^{\mathrm{Cyg}-\mathrm{X}-3} \approx 3.5 \times 10^{-3} \dot{N}_{\mathrm{p}, \mathrm{jet}}^{\mathrm{SS} 443}$. SS433 ejects hadrons in a quasi-steady fashion, whereas Cygnus X-3 is supposed to eject hadrons in a highly variable regime with a lower injection rate.

Our hypothesis for a hadronic interpretation of $\gamma$-ray emission from Cygnus X-3 needs to be supported by information that at the moment remains unavailable, such as hadronic emission lines in the flare spectra and a precise characterization of the $\gamma$-ray spectrum at energies below $100 \mathrm{MeV}$ that should show the characteristic decrement of neutral pion emission. Furthermore, hadronic mechanisms, besides emitting strong $\gamma$-ray radiation via $\pi^{0}$-decay, would produce an intense flux of high-energy neutrinos, emerging from the decay of secondary charged mesons produced in pp collisions. Hence, a firm simultaneous detection of strong neutrino flux and $\gamma$-ray activity from Cygnus X-3 would represent the signature of a dominant hadronic mechanism in the relativistic jet. In our hadronic scenario, owing to the temporal coincidence of the $\gamma$-ray/radio flares, we implicitly assume that the hadronic component of the jet provides the main contribution to the $\gamma$-ray emission, and the leptonic component produces - via synchrotron emission process - the strong radio flares far away from the compact object. In addition to $\gamma$-rays from $\pi^{0}$-decays, hadronic pp interactions are expected to produce a population of secondary electrons (and positrons) from the decay of charged pions $\left(\pi^{ \pm}\right)$. These secondary leptons can contribute to the emission in the radio band via synchrotron processes and in $\gamma$-rays (marginally with respect to the contribution by $\pi^{0}$-decay) via IC and bremsstrahlung processes.

\section{Conclusions}

Several events of $\gamma$-ray activity were detected by the AGILEGRID from Cygnus X-3 while the system was in a special radio/X-ray spectral state: intense $\gamma$-ray activity was detected during prominent minima of the hard X-ray light curve (corresponding to strong soft X-ray emission), a few days before intense radio outbursts (major radio flares). This temporal repetitive coincidence turned out to be the spectral signature of $\gamma$-ray activity from this puzzling microquasar, which might open new areas to study the interplay between the accretion disk, the corona, and the formation of relativistic jets. The simultaneous strong soft X-ray emission from the disk and $\gamma$-ray emission from the jet preceding the intense radio outbursts are consistent with a scenario in which the hot thermal corona "dissolves" and the accretion power from the disk directly charges the jet, emitting $\gamma$-rays and, subsequently, radio outbursts (via synchrotron processes) far from the compact object.

The $\gamma$-ray detections of Cygnus X-3 provide new constraints on emission models for this powerful X-ray binary, indicating that hybrid-Comptonization mechanisms (Coppi 1999) alone cannot account for the $\gamma$-ray fluxes detected by AGILE and Fermi above $100 \mathrm{MeV}$, unless we assume unrealistic physical parameters (Cerutti et al. 2011). This implies that the corona cannot be the site of the $\gamma$-ray emission. We found that the innermost part of the jet (distances $\lesssim 10^{10} \mathrm{~cm}$ from the compact object) could provide a strong contribution to the hard X-rays at $\sim 100 \mathrm{keV}$ during the $\gamma$-ray emitting interval, while the farthest part (distances $\gtrsim 10^{10} \mathrm{~cm}$ from the compact object) produces the bulk of the $\gamma$-ray emission above $100 \mathrm{MeV}$.

We found that the $\gamma$-ray spectrum of Cygnus X-3 detected by the AGILE-GRID is significantly harder than the time-averaged spectrum obtained by Fermi-LAT for the " $\gamma$-ray active periods" of the microquasar, lasting $\sim 4$ months (see Fig. A.2). Although both the AGILE main $\gamma$-ray events and the Fermi $\gamma$-ray active periods are both likely related to the presence of an active jet, the spectral difference may imply that there was a fast hardening of the spectrum during the peak $\gamma$-ray events, lasting $\sim 1-2$ days.

We have demonstrated that both a leptonic model based on inverse Compton emission from a relativistic plasmoid injected into the jet and a hadronic model based on $\pi^{0}$-decays, might account for the $\gamma$-ray emission observed by the AGILE-GRID. Both of these models require the introduction of a new component ("IC bump" or " $\pi^{0}$-bump") into the SED of the system. In both the leptonic and hadronic pictures, the inclination of the jet to the line of sight is assumed to be $i=14^{\circ}$.

A leptonic scenario seems to be more likely than a hadronic one: the $\gamma$-ray modulation, the spectral link between hard X-ray and $\gamma$-ray spectra, and the temporal link between $\gamma$-ray events and radio flares could be interpreted in a natural way by assuming that the electrons are the main emitters. According to our results, the HE $\gamma$-ray emission occurs at distances up to $\sim 10^{12} \mathrm{~cm}$ from the compact object. If we were to interpret the $\sim 4$-day delay between the onset of $\gamma$-ray and radio flaring emission as the propagation time of the relativistic jet $(v=\sqrt{5} c / 3)$, the radio burst would occur at a distance of $\sim 8 \times 10^{15} \mathrm{~cm}$. 
Table A.1. Main events of $\gamma$-ray emission detected by the AGILE-GRID in the period November 2007-July 2009.

\begin{tabular}{lccc}
\hline \hline Period & MJD & $\sqrt{\mathrm{TS}}$ & Flux $\left[10^{-8}\right.$ photons cm $\left.{ }^{-2} \mathrm{~s}^{-1}\right]$ \\
\hline 2008 Feb. 11 (18:07:28)-2008 Feb. 12 (11:07:44) & $54507.76-54508.46$ & 3.7 & $264 \pm 104$ \\
2008 Apr. 16 (13:59:12)-2008 Apr. 17 (13:48:00) & $54572.58-54573.58$ & 4.5 & $265 \pm 80$ \\
2008 Nov. 2 (13:01:05)-2008 Nov. 3 (19:01:05) & $54772.54-54773.79$ & 3.1 & $135 \pm 56$ \\
2008 Dec. 11 (19:50:40)-2008 Dec. 12(23:02:40) & $54811.83-54812.96$ & 4.0 & $190 \pm 65$ \\
2009 Jun. 20 (21:04:48)-2009 Jun. 21 (20:53:04) & $55002.88-55003.87$ & 3.8 & $193 \pm 67$ \\
2009 Jul. 13 (01:11:60)-2009 Jul. 14 (00:59:44) & $55025.05-55026.04$ & 3.2 & $216 \pm 89$ \\
2009 Jul. 21 (21:07:12)-2009 Jul. 23 (21:07:12) & $55033.88-55035.88$ & 3.6 & $158 \pm 59$ \\
\hline
\end{tabular}

Notes. All detections have a significance above $3 \sigma(\sqrt{T S} \geqslant 3)$. Column 1: period of detection in UTC; Col. 2: period of detection in MJD; Col. 3: significance of detection; Col. 4: photon flux (above $100 \mathrm{MeV}$ ).

Our hadronic model, with the assumption of a standard WR wind, would require a jet kinetic power of $L_{\text {kin, } \mathrm{p}} \approx 1.5 \times$ $10^{38} \mathrm{erg} \mathrm{s}^{-1}$ to explain the $\gamma$-ray emission detected by AGILE. This value is of the same order of magnitude as the bolometric luminosity of the disk/corona during the hypersoft spectral state, and lower than the Eddington accretion limit for a black hole with a mass of $M_{x} \approx 10 M_{\odot}\left(L_{\mathrm{Edd}} \approx 10^{39} \mathrm{erg} \mathrm{s}^{-1}\right)$. Thus, a hadronic picture is physically reasonable and not energetically less likely than a leptonic one. At present, there is no strong evidence that one of these hypotheses can be excluded, and it remains an open question whether the dominant process for $\gamma$-ray emission in microquasars is either hadronic or leptonic (Mirabel 2012).

The firm discovery of $\gamma$-ray emission from this microquasar represents the experimental proof that these astrophysical objects are capable of accelerating particles up to relativistic energies, through a mechanism - related to the disk-corona dynamics - that leads to jet formation.

Acknowledgements. The authors are grateful to the anonymous referee for her/his stimulating comments on the manuscript. We also thank A. Zdziarski for discussions about this work. This investigation was carried out with partial support under ASI contracts Nos. I/089/06/2, and I/042/10/0.

\section{Appendix A: The AGILE-GRID dataset}

We performed an analysis of the whole AGILE-GRID data in the period November 2007-July 2009 using a detection algorithm developed by the AGILE team to automatically search for transient $\gamma$-ray emission. The algorithm initially analyzed 140 maps, each related to a 2-day integration (non-overlapping consecutive time intervals). The time bins containing the peak $\gamma$-ray emission, with detection significances greater than $3 \sigma$, were identified. The analysis was subsequently manually refined to optimize the determination of the time interval of the $\gamma$-ray emission. The whole analysis was carried out with the Build 19 version of the AGILE team software, using the FM3.119_2 calibrated filter applied to the consolidated dataset with off-axis angles smaller than $40^{\circ}$. We used a multi-source maximum-likelihood analysis (MSLA) to take into account the emission of the nearby $\gamma$-ray pulsars ${ }^{16}$ 1AGL J2021+3652 (PSR J2021+3651), 1AGL J2022+4032 (PSR J2021+4026), and 1AGL J2032+4102 (PSR J2032+4127). In particular, the MSLA is fundamental to avoid contamination by the pulsar PSR J2032+4127, located

\footnotetext{
${ }^{16}$ The main characteristics of the persistent $\gamma$-ray sources that we used in the MSLA are reported in Table 1 of Chen et al. (2011).
}

at a distance of $\sim 0.5^{\circ}$. In this paper, we did not consider the off-pulse data for the nearby pulsar. Nevertheless, the MSLA accounted for the steady $\gamma$-ray emission from this source when calculating the significance and the flux of each $\gamma$-ray detection of Cygnus X-3. Moreover, we can exclude any substantial spectral contamination from the pulsar because the steady $\gamma$-ray emission from the pulsar ${ }^{17}$ is much fainter than the mean flux of the active $\gamma$-ray emission from Cygnus $\mathrm{X}-3$.

The main events of $\gamma$-ray activity, detected with a significance above $3 \sigma(\sqrt{T S} \geqslant 3)$, are shown in Table A.1. We found seven events ${ }^{18}$, including those presented in Tavani et al. (2009a) and Bulgarelli et al. (2012a).

By integrating all the main events with the FM3.119_2 filter, we detected a $\gamma$-ray source at $6.7 \sigma(\sqrt{T S}=6.7)$ at the average Galactic coordinate $(l, b)=\left(79.7^{\circ}, 0.9^{\circ}\right) \pm 0.4^{\circ}$ (stat) $\pm 0.1^{\circ}$ (syst), with a photon flux of $(158 \pm 29) \times 10^{-8}$ photons $\mathrm{cm}^{-2} \mathrm{~s}^{-1}$ above $100 \mathrm{MeV}^{19}$. The average differential spectrum between $100 \mathrm{MeV}$ and $3 \mathrm{GeV}$ is well-fitted by a power law with a photon index $\alpha=2.0 \pm 0.2$ (Fig. A.1). This value is consistent with the Cygnus X-3 photon index found by Tavani et al. (2009a) and Bulgarelli et al. (2012a). In Fig. A.2, we compare the $v F_{v}$ spectra of Cygnus X-3 obtained by the AGILE-GRID and Fermi-LAT (Abdo et al. 2009) during the $\gamma$-ray activity. We remark that the AGILE-GRID spectrum is related only to the peak $\gamma$-ray activity (the seven main events, lasting 1-2 days, in Table A.1), whereas the Fermi-LAT spectrum is an average spectrum found during the two active windows (of about two months each) of $\gamma$-ray emission from Cygnus X-3 (MJD: 54 750-54 820 and MJD: 54 990-55 045).

Finally, we evaluated the post-trial significance for repeated flare occurrences by using the same formalism of Bulgarelli et al. (2012b). The probability of having $k$ or more

\footnotetext{
17 The steady $\gamma$-ray emission from the pulsar PSR J2032+4127, as detected by the AGILE-GRID, is $F_{\gamma}^{\mathrm{PSR}}=[37 \pm 4$ (stat) $\pm 10 \%$ (syst) $] \times$ $10^{-8}$ photons $\mathrm{cm}^{-2} \mathrm{~s}^{-1}$ for photon energies above $100 \mathrm{MeV}$, see Chen et al. (2011) for details.

18 All the $\gamma$-ray events were detected by using the same filter, FM3.119_2. As discussed in the supplementary information of Tavani et al. (2009a), the event of 2-3 November 2008 appears to be relatively "soft" in $\gamma$-rays compared to the other episodes. By analyzing the event with the FT3ab_2 filter (which is more efficient in detecting this kind of emission), we found a more significant detection of $\sqrt{T S}=3.9$, at photon fluxes above $100 \mathrm{MeV}$ equal to $(214 \pm 73) \times 10^{-8}$ photons $\mathrm{cm}^{-2} \mathrm{~s}^{-1}$. ${ }^{19}$ Here we present an updated result for the analysis of the 7-event integration with respect to the one reported in Piano et al. (2011). In this paper, our analysis was carried out with a more recent version of the AGILE software tool (AG_multi4).
} 


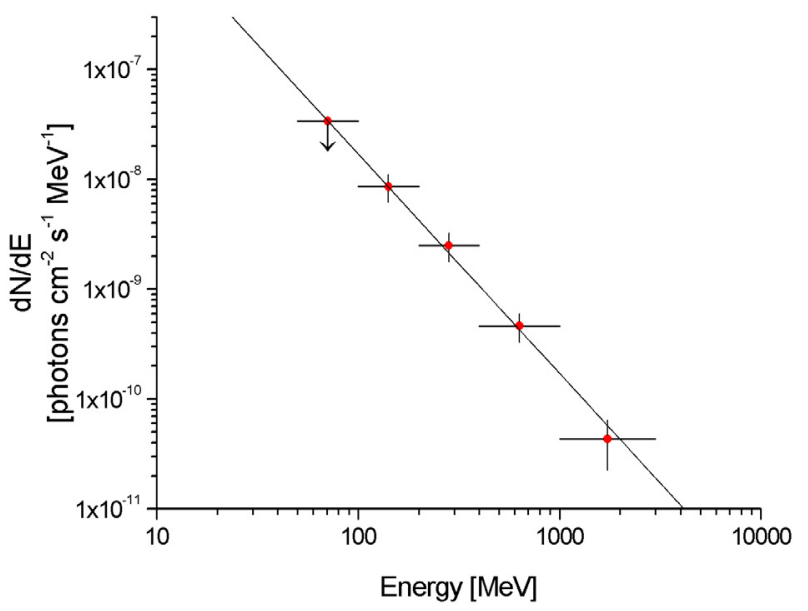

Fig. A.1. Photon spectrum between $50 \mathrm{MeV}$ and $3 \mathrm{GeV}$ of Cygnus X-3 found by the AGILE-GRID by integrating all the main $\gamma$-ray episodes in Table A.1. Power-law fit to $\gamma$-ray data between $100 \mathrm{MeV}$ and $3 \mathrm{GeV}$ with photon index $\alpha=2.0 \pm 0.2$.

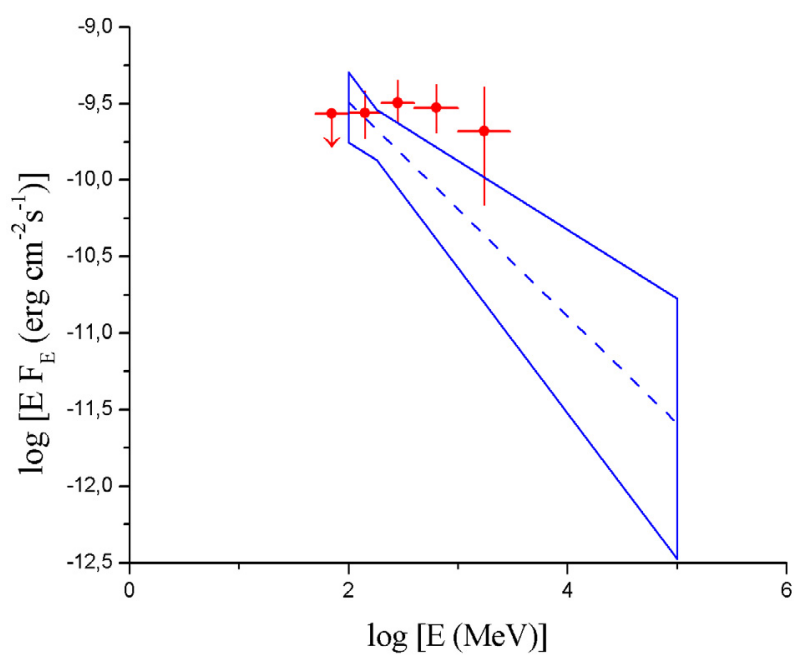

Fig. A.2. The $v F_{v}$ spectra of Cygnus X-3 during the $\gamma$-ray activity. Red circles: AGILE-GRID energy spectrum $(50 \mathrm{MeV}$ to $3 \mathrm{GeV})$ of the main episodes (Fig. A.1). Blue error contours and dashed blue line: average power-law fit with $\alpha=2.70 \pm 0.25$ of the spectrum obtained by Fermi-LAT integrating the two active windows of about two months each (Abdo et al. 2009).

detections - consistent with the position of Cygnus X-3 - with $\sqrt{T S} \geqslant \sqrt{h}$ in $N$ trials, is

$P(N, k)=1-\sum_{j=0}^{k-1}\left(\begin{array}{c}N \\ j\end{array}\right) p^{j}(1-p)^{N-j}$

where $p$ is the $p$-value corresponding to $h$. For $\sqrt{T S} \geqslant 3$, we have a $p$-value of $p=2.0 \times 10^{-3}$. Thus, for $N=140$ (our trials, the number of 2-day integration maps analyzed by the initial algorithm) and $k=7$ (our detections of Cygnus X-3), we found $P(140,7)=1.8 \times 10^{-8}$, which corresponds to 5.5 Gaussian standard deviations.

An MSLA applied to the deep integration of the AGILEGRID data (between November 2007 and July 2009) found weak persistent emission from a position consistent with
Cygnus X-3 $3^{20}$ (significance $\sqrt{T S}=5.17$ and photon flux $F_{\gamma}=(14 \pm 3) \times 10^{-8}$ photons $\left.\mathrm{cm}^{-2} \mathrm{~s}^{-1}\right)$.

\section{References}

Abdo, A. A., Ackermann, M., Ajello, M., et al. 2009, Science, 326, 1512 Aharonian, F. A., \& Atoyan, A. M. 1981, Ap\&SS, 79, 321

Albert, J., Aliu, E., Anderhub, H., et al. 2007, ApJ, 665, L51

Aleksić, J., Antonelli, L. A., Antoranz, P., et al. 2010, ApJ, 721, 843

Atoyan, A. M., \& Aharonian, F. A. 1999, MNRAS, 302, 253

Axelsson, M., Larsson, S., \& Hjalmarsdotter, L. 2009, MNRAS, 394, 1544

Barbiellini, G., Fedel, G., Liello, F., et al. 2002, Nucl. Instr. Meth. Phys. Res. A, 490, 146

Becklin, E. E., Neugebauer, G., Hawkins, F. J., et al. 1973, Nature, 245, 302

Bhat, C. L., Sapru, M. L., \& Razdan, H. 1986, ApJ, 306, 587

Bonnet-Bidaud, J. M., \& Chardin, G. 1988, Phys. Rep., 170, 325

Bulgarelli, A., Tavani, M., Chen, A. W., et al. 2012a, A\&A, 538, A63

Bulgarelli, A., Chen, A. W., Tavani, M., et al. 2012b, A\&A, 540, A79

Cattaneo, P. W., Argan, A., Boffelli, F., et al. 2011, Nucl. Instr. Meth. Phys. Res. A, 630, 251

Cerutti, B., Dubus, G., Malzac, J., et al. 2011, A\&A, 529, A120

Chen, A. W., Piano, G., Piano, G., Tavani, M., et al. 2011, A\&A, 525, A33

Coppi, P. S. 1999, High Energy Processes in Accreting Black Holes, 161, 375

Corbel, S., Dubus, G., Tomsick, J. A., et al. 2012 [arXiv: 1201.3356]

Danaher, S., Fegan, D. J., Porter, N. A., \& Weekes, T. C. 1981, Nature, 289, 568

Dubus, G., Cerutti, B., \& Henri, G. 2010, MNRAS, 404, L55

Fabrika, S. N., \& Borisov, N. V. 1987, Sov. Astron. Lett., 13, 279

Feroci, M., Costa, E., Soffitta, P., et al. 2007, Nucl. Instr. Meth. Phys. Res. A, 581,728

Giacconi, R., Gorenstein, P., Gursky, H., \& Waters, J. R. 1967, ApJ, 148, L119

Hanson, M. M., Still, M. D., \& Fender, R. P. 2000, ApJ, 541, 308

Hermsen, W., Bloemen, J. B. G. M., Jansen, F. A., et al. 1987, A\&A, 175, 141

H.E.S.S. Collaboration, Acero, F., Aharonian, F., \& Akhperjanian, A. G. 2009, A\&A, 508, 1135

Hjalmarsdotter, L., Zdziarski, A. A., Szostek, A., \& Hannikainen, D. C. 2009, MNRAS, 392, 251

Kelner, S. R., Aharonian, F. A., \& Bugayov, V. V. 2006, Phys. Rev. D, 74, 034018

Koljonen, K. I. I., Hannikainen, D. C., McCollough, M. L., Pooley, G. G., \& Trushkin, S. A. 2010, MNRAS, 406, 307

Koljonen, K. I. I., Hannikainen, D. C., \& McCollough, M. L. 2011, MNRAS, 416, L84

Konigl, A. 1983, MNRAS, 205, 471

Labanti, C., Marisaldi, M., Fuschino, F., et al. 2006, Proc. SPIE, 6266, 110

Lamb, R. C., Godfrey, C. P., Wheaton, W. A., \& Tumer, T. 1982, Nature, 296, 543

Ling, Z., Zhang, S. N., \& Tang, S. 2009, ApJ, 695, 1111

McCollough, M. L., Robinson, C. R., Zhang, S. N., et al. 1999, ApJ, 517, 951

Migliari, S., Fender, R., \& Méndez, M. 2002, Science, 297, 1673

Mioduszewski, A. J., Rupen, M. P., Hjellming, R. M., Pooley, G. G., \& Waltman, E. B. 2001, ApJ, 553, 766

Mirabel, I. F. 2012, Science, 335, 175

Mori, M., Bertsch, D. L., Dingus, B. L., et al. 1997, ApJ, 476, 842

O'Flaherty, K. S., Cawley, M. F., Fegan, D. J., et al. 1992, ApJ, 396, 674

Parsignault, D. R., Gursky, H., Kellogg, E. M., et al. 1972, Nature, 239, 123

Perotti, F., Fiorini, M., Incorvaia, S., Mattaini, E., \& Sant'Ambrogio, E. 2006, Nucl. Instr. Meth. Phys. Res. A, 556, 228

Piano, G., Bulgarelli, A., Tavani, M., et al. 2011 [arXiv: 1110.6043]

Pittori, C., Verrecchia, F., Verrecchia, F., Chen, A. W., et al. 2009, A\&A, 506, 1563

Prest, M., Barbiellini, G., Bordignon, G., et al. 2003, Nucl. Instr. Meth. Phys. Res. A, 501, 280

Reynoso, M. M., Romero, G. E., \& Christiansen, H. R. 2008, MNRAS, 387, 1745

Romero, G. E., Torres, D. F., Kaufman Bernadó, M. M., \& Mirabel, I. F. 2003, A\&A, 410, L1

Romero, G. E., Christiansen, H. R., \& Orellana, M. 2005, ApJ, 632, 1093

Samorski, M., \& Stamm, W. 1983, ApJ, 268, L17

Shrader, C. R., Titarchuk, L., \& Shaposhnikov, N. 2010, ApJ, 718, 488

Stark, M. J., \& Saia, M. 2003, ApJ, 587, L101

Szostek A., \& Zdziarski A. A. 2008, MNRAS, 386, $593 \mathrm{~S}$

Szostek, A., Zdziarski, A. A., \& McCollough, M. L. 2008, MNRAS, 388, 1001

Tavani, M., Bulgarelli, A., Piano, G., et al. 2009a, Nature, 462, 620

Tavani, M., Barbiellini, G., Argan, A., et al. 2009b, A\&A, 502, 995

20 The persistent AGILE-GRID source associated to Cygnus X-3 is $\mathrm{J} 2033+4050$ in Table 1 of Chen et al. (2011). 
Tudose, V.,Fender, R. P., Garrett, M. A., et al. 2007, MNRAS, 375, L11 Tudose, V., Miller-Jones, J. C. A., Fender, R. P., et al. 2010, MNRAS, 401, 890 van Kerkwijk, M. H., Charles, P. A., Geballe, T. R., et al. 1992, Nature, 355, 703

Vilhu, O., Hakala, P., Hannikainen, D. C., McCollough, \& M., Koljonen, K. 2009, A\&A, 501, 679

Vladimirsky, B. M., Stepanian, A. A., \& Fomin, V. P. 1973, ICRC, 1, 456

Zdziarski, A. A., Sikora, M., Dubus, G., et al. 2012a, MNRAS, 421, 2956

Zdziarski, A. A., Maitra, C., Frankowski, A., Skinner, G. K., \& Misra, R. 2012b, MNRAS, in press [arXiv: 1205.4402]

1 INAF/IAPS, via del Fosso del Cavaliere 100, 00133 Roma, Italy e-mail: giovanni.piano@iaps.inaf.it

2 CIFS-Torino, viale Settimio Severo 3, 10133 Torino, Italy

3 Dipartimento di Fisica, Università di Roma "Tor Vergata", via della Ricerca Scientifica 1, 00133 Roma, Italy

4 INAF/IASF-Milano, via E. Bassini 15, 20133 Milano, Italy

5 INAF/IASF-Bologna, via Gobetti 101, 40129 Bologna, Italy

${ }^{6}$ Dipartimento di Fisica and INFN Trieste, via Valerio 2, 34127 Trieste, Italy

7 INFN-Pavia, via Bassi 6, 27100 Pavia, Italy

8 INFN-Roma "Tor Vergata", via della Ricerca Scientifica 1, 00133 Roma, Italy
9 INAF - Osservatorio Astronomico di Cagliari, località Poggio dei Pini, strada 54, 09012 Capoterra, Italy

10 ENEA Frascati, via E. Fermi 45, 00044 Frascati, Roma, Italy

11 ASI Science Data Center (ASDC), via G. Galilei, 00044 Frascati, Roma, Italy

12 INAF-OAR, via Frascati 33, 00040 Monte Porzio Catone, Italy

13 University of the Witwatersrand, School of Physics, WITS 2050 Johannesburg, South Africa

14 INAF - IASF - Palermo, via U. La Malfa 15, 90146 Palermo, Italy

15 Department of Astronomy, Yale University, PO Box 208101, New Haven, CT 06520-8101, USA

16 Aalto University Metsähovi Radio Observatory, Metsähovintie 114 02540 Kylmälä, Finland

17 Department of Physics and Space Sciences, Florida Institute of Technology, 150 W. University Blvd., Melbourne, FL 32901, USA

18 Smithsonian Astrophysical Observatory, 60 Garden Street, Cambridge, Massachusetts 02138, USA

19 Astrophysics Group, Cavendish Laboratory, 19 J. J. Thomson Avenue, Cambridge CB3 OHE, UK

20 Special Astrophysical Observatory RAS, Karachaevo-Cherkassian Republic, 369169 Nizhnij Arkhyz, Russia

21 Departament d'Astronomia i Meteorologia, Institut de Ciències del Cosmos, Facultat de Física, 7a planta, Universitat de Barcelona Martí i Franquès 1, 08028 Barcelona, Spain 